\title{
REQUIRED REDUNDANCY IN THE REPRESENTATION OF REALS
}

\author{
MICHAEL STARBIRD AND THOMAS STARBIRD
}

(Communicated by Andrew M. Bruckner)

\begin{abstract}
Redundancy in decimal-like representations of reals cannot be avoided. It is proved here that if $\left\{A_{i}\right\}_{i=0,1,2, \ldots}$ is a countable collection of countable (or finite) sets of reals such that for each real $x$ there are $a_{i} \in A_{i}$ with

$$
x=\sum_{i=0}^{\infty} a_{i},
$$

then there is a dense subset of reals with redundant representations; that is, there is a dense set $C$ of $\mathbb{R}$ such that for each $x$ in $C, x=\sum_{i=0}^{\infty} a_{i}$ and $x=\sum_{i=0}^{\infty} b_{i}$ with $a_{i}, b_{i}$ in $A_{i}$, but $a_{i} \neq b_{i}$ for some $i$. Petkovsek [1] proved a similar result under the added assumption that every sum of the form $\sum_{i=0}^{\infty} a_{i}$ with $a_{i} \in A_{i}$ converges.
\end{abstract}

\section{INTRODUCTION}

Every real number can be expressed as a decimal expansion, and each decimal expansion is shorthand for the limit of a convergent series. Let $A_{0}=\mathbb{Z}$, and for each $i=1,2, \ldots$ let $A_{i}=\left\{0,10^{-i}, 2 \times 10^{-i}, \ldots, 9 \times 10^{-i}\right\}$. Then each real number can be written as $\sum_{i=0}^{\infty} a_{i}$ where $a_{i} \in A_{i}$. Sometimes it is annoying that certain numbers have two different representations as such infinite series, e.g., $.19999 \ldots=.2000 \ldots$.

One might hope that it would be possible to eliminate this annoying redundancy by choosing different sets of numbers $A_{i}$. For example, we might let $A_{1}=\{\pi / 4, e / 7,34,-21 / 3\}$, and let the other $A_{i}$ 's be equally unlikely sets of reals including negative numbers and large numbers. We would insist only that every real number is the limit of some series $\sum_{i=0}^{\infty} a_{i}$ and $a_{i}$ is in $A_{i}$.

The standard representation of numbers has the added property that for every choice of $a_{i} \in A_{i}, \sum_{i=0}^{\infty} a_{i}$ does in fact converge. Representations of reals in which each $\sum_{i=0}^{\infty} a_{i}$ with $a_{i} \in A_{i}$ converges were considered in [1]. Techniques in [1] show that if $\left\{A_{i}\right\}$ is a collection of finite sets of reals such that every $\sum_{i=0}^{\infty} a_{i}$ with $a_{i} \in A_{i}$ converges, and every real in some interval is of the form $\sum_{i=0}^{\infty} a_{i}$ with $a_{i} \in A_{i}$, then the set of numbers with redundant representations is dense in the interval. Basically the proof is to note that the function $f: \Pi A_{i} \rightarrow$ $\mathbb{R}$ defined by $f\left(a_{0}, a_{1}, \ldots\right)=\sum_{i=0}^{\infty} a_{i}$ is a continuous function from a Cantor

Received by the editors September 28, 1990.

1980 Mathematics Subject Classification (1985 Revision). Primary 40A05.

Key words and phrases. Ambiguous numbers, redundant representations. 
set over $\mathbb{R}$. If it were one-to-one any interval, then that interval would be homeomorphic to a subset of a Cantor set, which is not true.

In fact, even without the assumption that every $\sum_{i=0}^{\infty} a_{i}$ with $a_{i} \in A_{i}$ converges, it remains true that reals with redundant representations are dense.

Our theorem is the following.

Theorem. Let $\left\{A_{i}\right\}_{i=0,1,2, \ldots}$ be a countable collection of countable (or finite) sets of reals such that for each real $x$ there are $a_{i} \in A_{i}$ with $x=\sum_{i=0}^{\infty} a_{i}$. Then there is a dense subset of reals with redundant representations, that is, there is a dense set $C$ of $\mathbb{R}$ such that for each $x$ in $C, x=\sum_{i=0}^{\infty} a_{i}$ and $x=\sum_{i=0}^{\infty} b_{i}$, with $a_{i}, b_{i}$ in $A_{i}$, but $a_{i} \neq b_{i}$ for some $i$.

\section{DEFINITIONS AND NOTATION}

For each $i=0,2, \cdots, A_{i}$ is a finite or countably infinite set of real numbers. An $A$-segment is a finite sequence $a_{0}, \ldots, a_{n-1}$ with $a_{i} \in A_{i}, i=0,1,2$, $\ldots, n-1$. Its sum is $\sum_{i=0}^{n-1} a_{i}$, and its length is $n$. Sometimes we denote an $A$-segment and its sum by the same letter (usually $P$ or $Q$ ).

An $A$-series is an infinite sequence $a_{0}, a_{1}, \ldots$ with $a_{i} \in A_{i}$ for $i=0,1$, $2, \ldots$. Its sum is the infinite sum $\sum_{i=0}^{\infty} a_{i}$, which exists only when the series converges. Its length is infinite. The assumption in the theorem is that every real number is the sum of some $A$-series.

One $A$-segment or $A$-series $Q$ is an extension of an $A$-segment $P$ if length $Q>$ length $P$, and the first length $P$ elements of $Q$ equal the corresponding elements of $P$.

In the proof of the theorem, we will construct two $A$-segments $P$ and $Q$ with the same length but different sums (so they must be distinct), but that have $A$-series extensions with equal sums $x$. Thus, $x$ has two distinct $A$ series representations.

Denote by $T(N)$ the $N$ th tail set, which is the set of all limits of convergent series $\sum_{i=N}^{\infty} a_{i}$ with $a_{i} \in A_{i}$. A compact way to state the assumption of the theorem is $T(0)=\mathbb{R}$.

$T(N, \mu)$, the $N$ th tails of $\mu$-bounded deviation, is the subset of $T(N)$ with representations whose partial sums obey

$$
\left|\sum_{i=N}^{M} a_{i}\right|<\mu \quad \text { for all } M
$$

The tail sets are of interest because the sum of an $A$-segment $P$ added to all the numbers in the tail set $T$ (length $P$ ) gives all the reals expressible by $A$-series extensions of $P$. This set of reals is called the leaves of $P$.

Similarly, the $\mu$-leaves of $P$, the set of which is denoted Leaves $(P, \mu)$, are all reals equal to the sum of $P$ added to all the numbers in $T$ (length $P, \mu$ ). Leaves $(P, \mu)$ play a crucial role in the proofs. The $\mu$-boundedness gives control over series that we build, leading to convergence.

For a set $D$ of real numbers, $\operatorname{cl}(D)$ denotes the closure of $D$. 


\section{BASIC LEMMAS AND PROPOSITIONS}

Our first lemma states that the $\mu$-tails are not too sparse.

Lemma 1. Assume $T(0)=\mathbb{R}$. Given $\mu>0$, there exists $N$ such that for all $i \geq N, \operatorname{cl}(T(i, \mu))$ contains an open interval (which depends on $i$ ).

Proof of Lemma 1. We prove Lemma 1 by writing $\mathbb{R}$ as a countable union of sets and then applying the Baire Category Theorem.

Each real number $x$ is the sum of an $A$-series $\sum_{i=0}^{\infty} a_{i}$, so for each $x$ there is a number $N(x)$ such that $\left|\sum_{i=m}^{n} a_{i}\right|<\mu / 4$ for all $n \geq m>N(x)$. Thus for all $m>N(x), x \in \operatorname{Leaves}(P, \mu / 4)$ for some $A$-segment $P$ of length $m$. Thus for any $N$,

$$
\mathbb{R}=\bigcup_{k=N}^{\infty} \cup\{\operatorname{Leaves}(P, \mu / 4): P \text { is an } A \text {-segment of length } k\} .
$$

Since there are only countably many $A$-segments of any given finite length, this double union is a countable union. By the Baire Category Theorem, for some $k$ and some $A$-segment $P$ of length $k, \operatorname{cl}($ Leaves $(P, \mu / 4))$ contains an open interval. Since $\operatorname{Leaves}(P, \mu / 4)$ is a translate of $T(k, \mu / 4), \operatorname{cl}(T(k, \mu / 4))$ contains an open interval. Since $N$ is arbitrary in the union, there is a sequence $k_{1}<k_{2}<k_{3}<\ldots$ such that for each $i=1,2, \ldots, \operatorname{cl}\left(T\left(k_{i}, \mu / 4\right)\right)$ contains an open interval.

Let $n$ be an integer with $n>k_{1}$. We now show that $\operatorname{cl}(T(n, \mu))$ contains an open interval.

Let $y \in T\left(k_{1}, \mu / 4\right)$. Then $y=\sum_{i=k_{1}}^{\infty} a_{i}$ with $a_{i} \in A_{i}$ where $\left|\sum_{i=k_{1}}^{m} a_{i}\right|<$ $\mu / 4$ for all $m \geq k_{1}$. Therefore $\left|\sum_{i=n}^{m} a_{i}\right|<\mu / 2$ for any $m \geq n$. Choose $j$ with $n<k_{j}$. Then for each $x \in T\left(k_{j}, \mu / 4\right), \sum_{i=n}^{k_{j}-1} a_{i}+x$ is a point in $T(n, 3 \mu / 4)$. Since $\operatorname{cl}\left(T\left(k_{j}, \mu / 4\right)\right)$ contains an interval, $\operatorname{cl}(T(n, 3 \mu / 4))$, and therefore, $\operatorname{cl}(T(n, \mu))$ contains an interval.

Lemma 2. Assume $T(0)=\mathbb{R}$. Suppose $P$ is an A-segment, $\mu>0, I$ is a nondegenerate closed interval, and

$$
\operatorname{cl}(\operatorname{Leaves}(P, \mu)) \supset I \text {. }
$$

Then given $\delta>0$, there exists a $\mu$-extension $Q$ of $P$ and a nondegenerate closed interval $J \subset I$ such that

$$
\operatorname{cl}(\text { Leaves }(Q, \delta)) \supset J .
$$

Proof of Lemma 2. Let $\lambda=\min (|I| / 3, \delta)$. By Lemma 1 , there is an $N$ such that for $i>N, \operatorname{cl}(T(i, \lambda))$ contains a nondegenerate closed interval $K(i)$. Let $x$ be in the middle third of $I$ and in Leaves $(P, \mu)$. $\mu$-extend $P$ to $Q$ such that $Q$ approximates $x$ sufficiently well so that $Q$ 's sum is in the middle third of $I$, and length $Q>N$. Then $\operatorname{cl}($ Leaves $(Q, \lambda))$ contains a translate $J$ of $K($ length $Q)$ (and so surely $\operatorname{cl}(\operatorname{Leaves}(Q, \delta)) \supset J)$. Since $\operatorname{cl}(\operatorname{Leaves}(Q, \lambda)) \subset I$, $J \subset I$.

Our next proposition is a key in deriving the fact that a number has two representations.

Proposition 1. Assume $T(0)=\mathbb{R}$. Let $P$ and $Q$ be A-segments with $\operatorname{cl}($ Leaves $(P, 1))$ and $\operatorname{cl}($ Leaves $(Q, 1))$ both containing an open interval $I$. Then there is a real number in $\operatorname{Leaves}(P, 1) \cap \operatorname{Leaves}(Q, 1)$. 
Proof of Proposition 1. Using Lemma 2, extend $P$ to $P^{\prime}$ such that $\operatorname{cl}\left(\right.$ Leaves $\left.\left(P^{\prime}, 1 / 2\right)\right)$ contains a closed interval $J$ in $I$. Then using the lemma again, extend $Q$ to $Q^{\prime}$ such that $\operatorname{cl}\left(\right.$ Leaves $\left.\left(Q^{\prime}, 1 / 2\right)\right)$ contains a closed interval $I^{\prime}$ in $J$. Thus both $\mathrm{cl}\left(\operatorname{Leaves}\left(P^{\prime}, 1 / 2\right)\right)$ and $\mathrm{cl}\left(\operatorname{Leaves}\left(Q^{\prime}, 1 / 2\right)\right)$ contain $I^{\prime}$.

Now repeat the above steps, getting $\frac{1}{2}$-extensions $P^{\prime \prime}, Q^{\prime \prime}$, and a closed subinterval $I^{\prime \prime}$ of $I^{\prime}$, with $\operatorname{cl}\left(\operatorname{Leaves}\left(P^{\prime \prime}, 1 / 4\right)\right)$ and $\operatorname{cl}\left(\operatorname{Leaves}\left(Q^{\prime \prime}, 1 / 4\right)\right)$ both containing $I^{\prime \prime}$. Continuing in this way gives a sequence of nested intervals converging to a point, and a sequence of further and further extensions $P$, $P^{\prime}, P^{\prime \prime}, \ldots$ that form an $A$-series converging to that point. But $Q, Q^{\prime}, Q^{\prime \prime}, \ldots$ also form an $A$-series converging to the same point. This completes the proof of Proposition 1.

\section{PROOF OF THE THEOREM}

The proof of the theorem divides into two cases, depending on whether or not there is a nonzero "offset."

Definition. An offset is a number that is the limit of numbers $t_{n_{i}} \in T\left(n_{i}\right)$ where $n_{1}<n_{2}<n_{3}<\cdots$.

When a nonzero offset exists, the natural way to construct a series designed to converge to a certain value may yield a series that does not converge because it continually jumps. The following example illustrates this technical difficulty.

Example. Let

$$
\begin{aligned}
& B_{1}=\{0,0.1,0.2,0.3, \ldots, 0.8\}, \\
& B_{2}=\{0,-2\}, \\
& B_{3}=\{0,2.1\}, \\
& B_{4}=\{0,0.01,0.02,0.03, \ldots, 0.08\}, \\
& B_{5}=\{0,-2\}, \\
& B_{6}=\{0,2.01\},
\end{aligned}
$$

and so on. Note that 1 is a limit point of the set of numbers to which convergent series with terms from the $B_{j}$ 's converge, but 1 is not itself the sum of such a convergent series. The natural way of attempting to produce a series converging to 1 would contain infinitely many -2 's.

In this example, -2 is an offset, as is 2 . In the ordinary decimal system where $A_{i}$ is $\left\{0,10^{-i}, 2 \times 10^{i}, \ldots, 9 \times 10^{-i}\right\}$, the diameter of the tails $T(i)$ converges to 0 as $i \rightarrow \infty$, so there is no offset except 0 .

First we deal with the case where a nonzero offset exists.

Proposition 2. Assume that $T(0)=\mathbb{R}$. If there is an offset $d \neq 0$, then the set of real numbers that are sums of more than one A-series is dense in $\mathbb{R}$.

Proof. The aim of this proof is to construct two $A$-series with the same sum. One has partial sums that do not vary much after $N$ terms, while the other incorporates a variation of approximately $d$.

Let $I$ be an open interval. We will produce a point in $I$ that is the sum of more than one $A$-series. We assume without loss of generality that the length of $I$ is less than $|d| / 2$.

Apply Lemma 1 with $\mu=|I| / 3$. There exists $L$ such that for $i>L$, $\operatorname{cl}(T(i, \mu))$ contains an open interval. 
Let $Q$ be an $A$-segment of length $M>L$ whose sum is in the middle third of $I$. Then $\operatorname{cl}($ Leaves $(Q, \mu))$ contains some interval $J \subset I$. Let $y$ be the midpoint of $J$. By Lemma 1 there exists $M^{\prime}>M$ such that for $i>M^{\prime}$, $\operatorname{cl}(T(i,|J| / 3))$ contains an open interval.

Since $T(0)=\mathbb{R}$, there is an $A$-series $P$ converging to $y-d$. Find an initial segment $P^{N}$ of $P$ of length $N>M^{\prime}$ such that for $n \geq N$,

$$
\left|P^{n}-(y-d)\right|<|J| / 12 .
$$

We claim that $P^{N}$ has a finite extension $P^{\prime}$ whose sum is in the middle third of $J$. To find one, use the definition of offset to find an $i>N$ and $d^{\prime} \in T(i)$ such that $\left|d-d^{\prime}\right|<|J| / 12$. Let $P^{\prime}$ be the first $i$ terms of $P$ extended by sufficiently many terms of $d^{\prime}$. Notice that $\mathrm{cl}\left(\operatorname{Leaves}\left(P^{\prime},|J| / 3\right)\right)$ contains some interval $K \subset J$, so $\operatorname{cl}\left(\operatorname{Leaves}\left(P^{\prime}, \mu\right)\right)$ and $\operatorname{cl}(\operatorname{Leaves}(Q, \mu))$ both contain $K$. Proposition 1 then implies that there is a number $x$ that is a $\mu$-leaf of both $P^{\prime}$ and $Q$. Since $x \in \operatorname{Leaves}(Q, \mu), x$ is the sum of an $A$-series whose partial sums (of more than $M$ terms) are all in $I$, unlike the sum of $P^{N}$, which is close to $(y-d)$ and is therefore not in $I$. Thus these two representations of $x$ are different.

We now prove the theorem in the case where there is no nonzero offset.

Proposition 3. Assume that $T(0)=\mathbb{R}$. If there is no nonzero offset, then the set of real numbers that are sums of more than one A-series is dense in $\mathbb{R}$.

Proof. Let $I$ be an open interval. We will produce a point in $I$ that is the sum of more than one $A$-series.

Choose $\mu<|I| / 6$. By Lemma 1 there is an integer $N$ such that for $i>N$, $\operatorname{cl}(T(i, \mu))$ contains an open interval. Let $Q$ be an $A$-segment of length $M>$ $N$ whose sum is in the middle third of $I$. Then $\operatorname{cl}(\operatorname{Leaves}(Q, \mu))$ contains a nondegenerate closed interval $J \subset I$.

We will now show that there are different $\mu$-extensions $Q^{\prime}$ and $Q^{\prime \prime}$ of $Q$ such that Leaves $\left(Q^{\prime}, 2 \mu\right)$ and Leaves $\left(Q^{\prime \prime}, 2 \mu\right)$ both contain the same single point $x$.

Consider the $\mu$-leaves of $Q$ that are in $J$ and the corresponding $A$-series for each. For a given $i \geq$ length $Q$, the set of these $\mu$-leaves is naturally partitioned by the choice of $a \in A_{i}$ that appears in the series for a leaf. In other words, for each $a \in A_{i}$ we consider the subset $P_{a}$ of the $\mu$-leaves of $Q$ in $J$ that use $a$ as the $i$ th element in their series. Certainly there exists an $i \geq$ length $Q$ such that this partition is nondegenerate, i.e., with 2 or more different $P_{a}$ 's nonempty. For each such $i$, either there is only a finite number of $P_{a}$ 's nonempty or an infinite number. We now consider separately two cases, depending on whether there are any $i$ 's with infinitely many $P_{a}$ 's nonempty.

Case 1 . Suppose for every $i$, only finitely many $P_{a}$ 's are nonempty. Take the first $i \geq$ length $Q$ when 2 or more $P_{a}$ 's are nonempty. Since only a finite number of $P_{a}$ 's are nonempty,

$$
J=\operatorname{cl}\left(\bigcup\left\{P_{a}: a \in A_{i}\right\}\right)=\bigcup\left\{\mathrm{cl}\left(P_{a}\right): a \in A_{i}\right\} ;
$$

so there are $a^{\prime} \neq a^{\prime \prime}$, both in $A_{i}$, with $\operatorname{cl}\left(P_{a^{\prime}}\right) \cap \mathrm{cl}\left(P_{a^{\prime \prime}}\right) \neq \phi$ since $J$ is an interval. Let $x$ be a number in the intersection. Let $Q^{\prime}$ and $Q^{\prime \prime}$ denote 2 extensions of $Q$, both of length $i+1$ (i.e., both ending with the $i$ th term), using $a^{\prime}$ and 
$a^{\prime \prime}$, respectively, as their $i$ th term. The point $x$, being in $\operatorname{cl}(\operatorname{Leaves}(Q, \mu))$, is in $\operatorname{cl}\left(\right.$ Leaves $\left.\left(Q^{\prime}, 2 \mu\right)\right)$ and $\operatorname{cl}\left(\right.$ Leaves $\left.\left(Q^{\prime \prime}, 2 \mu\right)\right)$.

Next we show that a standard diagonalization argument proves that $x$ is actually in Leaves $\left(Q^{\prime}, 2 \mu\right)$ and in Leaves $\left(Q^{\prime \prime}, 2 \mu\right)$. The proof is the same for both, so we give it only for $Q^{\prime}$. We build a candidate series and then show that it converges to $x$.

Find $y_{j} \in \operatorname{Leaves}\left(Q^{\prime}, 2 \mu\right)$ with $y_{j}$ converging to $x$. Infinitely many of the $y_{j}$ 's have the same $i+1$ term (more precisely, the representations that show that $y_{j} \in \operatorname{Leaves}\left(Q^{\prime}, 2 \mu\right)$ have the same $i+1$ term); so discard (for purposes of this paragraph only) any $y_{j}$ 's with different $i+1$ term, and set $b_{1}$ to that $i+1$ term. Now, of the $y_{j}$ 's remaining, infinitely many have the same $i+2$ term; so discard other $y_{j}$ 's, and set $b_{2}$ to that $i+2$ term. Continuing in this way, we generate a sequence $b_{j} \in A_{j}, j>i$. Let $P$ be the $A$-series that begins with $Q^{\prime}$ and then continues with the $b_{j}$ 's. We now claim that $P$ converges to $x$.

Since $y_{j} \in$ Leaves $\left(Q^{\prime}, 2 \mu\right)$, every partial sum (beyond length $Q^{\prime}$ ) of every $y_{j}$ is between $Q^{\prime}-2 \mu$ and $Q^{\prime}+2 \mu$. Any partial sum of $P$ is the partial sum of some $y_{j}$ (actually of infinitely many $y_{j}$ 's); so every partial sum of $P$ also lies between $Q^{\prime}-2 \mu$ and $Q^{\prime}+2 \mu$. Therefore, any subsequence of the partial sums of $P$ has a convergent subsequence. If any subsequence converged to $z \neq x$, then denoting the sum of the first $n$ terms of a series by superscript $n$,

$$
P^{n_{i}} \rightarrow S z
$$

and each $P^{n_{i}}$ agrees with some $y_{j}$, call it $y_{i}^{*}$, through $n_{i}$ terms. As a result,

$$
y_{i}^{*}=y_{i}^{n_{i}}+t_{i}
$$

where $t_{i} \in T\left(n_{i}, 4 \mu\right) . y_{i}^{*} \rightarrow x$, but $y_{i}^{n_{i}} \rightarrow z$, so $t_{i} \rightarrow x-z$, which contradicts the assumption that no nonzero offset exists. This concludes the proof of this case.

Case 2. Suppose that for some $i$, an infinite number of $P_{a}$ 's are nonempty.

Then, since all of these $a$ 's must be less than $2 \mu$ in absolute value, there is a cluster point of $a$ 's. We know that $\operatorname{cl}(T(i, \mu))$ contains some interval (Lemma 1) (which might be very short). Then $Q^{\prime}+\operatorname{cl}(T(i, \mu))$ contains some interval $K$, which must be in $l$ (since $\mu$ is a small fraction of $|I|)$. In $Q^{\prime}$, replacing its $i$ th term by two other $a$ values that are closer together than the length of $K$, giving $Q^{\prime \prime}$ and $Q^{\prime \prime \prime}$, we have that

$$
Q^{\prime \prime}+\operatorname{cl}(T(i, \mu)) \quad \text { and } \quad Q^{\prime \prime \prime}+\operatorname{cl}(T(i, \mu))
$$

both contain an interval in $I$, and the intervals overlap. By Proposition 1, there is a common $\mu$-leaf.

This completes the proof of the theorem.

\section{REFERENCES}

1. M. Petkovsek, Ambiguous numbers are dense, Amer. Math. Monthly, 97 (1990), 408-411.

Department of Mathematics, The University of Texas at Austin, Austin, Texas 78712

Jet Propulsion laboratory, California Institute of Technology, Pasadena, CaliforNIA 91109 